\title{
Nanocrystalline AlNiCoFeCrTi high-entropy alloy resulted from mechanical alloying and annealing
}

\author{
Alexandra Yurkova, Vadym Chernyavsky, Dmytro Hushchyk, Igor Bilyk \\ Igor Sikorsky Kyiv Polytechnic Institute, Kyiv, Ukraine \\ yurkova2403@gmai.com; vadikv13@gmail.com; dima94g@ukr.net; $\underline{\text { ii_bilyk@ukr.net }}$
}

\begin{abstract}
This study reports the structural evolution of multicomponent AlNiCoFeCrTi high-entropy alloy from elemental materials to nanocrystalline metastable solid solution during mechanical alloying (MA), and further, to equilibrium phases during subsequent thermal annealing. It was justified experimentally that shot-time mechanical milling of Al-Ni-Co-FeCr-Ti powder mixture during 3 hours resulted in a single-phase nanocrystalline high-entropy alloy (HEA) with a structure of bcc solid solution. During thermal annealing of the bcc solid solution phase transformation take place, and grain growth of equilibrium phases occur. The phase composition of as-MA alloy transforms to fec and bec solid solutions with $\mathrm{TiC}$ precipitations' when the MA powder was annealed at $1200{ }^{\circ} \mathrm{C}$ for $1 \mathrm{~h}$. X-ray diffraction and electron microscopy data show that the nanocrystalline powder microstructure is retained in the alloy with no grain growth. The AINiCoFeCrTi HEA exhibit 7.1 GPa and 9.2 $\pm 0.3 \mathrm{GPa}$ in Vickers hardness after mechanical alloying and after thermal annealing, respectively.
\end{abstract}

Keywords - high-entropy alloy; mechanical alloying; nanostructure; annealing; phase transformation; microhardness

\section{INTRODUCTION}

The conventional alloy design strategy involved adding one or more elements to a "host" metal to achieve enhancement in desired properties. Alloys traditionally have been based on a solvent element to which various solute atoms are added for improving specific properties. As a new focus on materials research and a novel alloy design concept, high entropy alloys (HEAs) have been achieved successfully by changing or designing the component elements of metallic material and have attracted great attentions of material researchers since it was proposed in 2004 [1,2]. HEAs are a new generation alloys and are quite different from traditional alloys, which are based on one or two elements. HEAs are typically defined as multicomponent alloys whose principal elements are at least five in equiatomic or near equiatomic ratio, and the concentration of each constituent element is between 5 and 35 at.\% [1-5]. They tend to exhibit solid solution structures, instead of complex phases, stabilized by their high configurational entropy of mixing [3-6]

HEAs have been studied extensively in recent years, and they are expected to be abundant in academic research and industrial applications. Some core effects in HEAs would be more pronounced than those in conventional alloys [1-4]: high entropy - it enhances the formation of simple solid solution phases, such as bcc and/or fcc structures, and thus simplifies the microstructures; severe lattice distortion - it arises from the atomic size difference among different elements in solid solution phases and might markedly influence mechanical, physical and chemical properties; sluggish diffusion - it is due to the inefficient cooperative diffusion of various species and thus slow down phase transformations; and cocktail - it comes from the ideal mixing and inevitably excess interactions among the multi-principal elements in solid solution phases, and thus is a composite effect on properties.

HEAs have more excellent properties than conventional alloys and exhibit promising future for engineering applications due to their good thermal stability, high hardness and high strength, excellent wear resistance, superior oxidization and corrosion resistance, as well as many other outstanding properties [3-8]. The promising properties of HEAs offer the potential to be used in many applications, such as tools, molds, dies, mechanical parts and furnace parts which require high strength, thermal stability and wear and oxidation resistance. These alloys can be used for various applications that demand high temperature strength, oxidation, corrosion and wear resistance [3,4,6,7].

For fabricating HEAs, three main routes are also applied: ingot metallurgy, powder metallurgy, and coating and deposition [4,5]. Most of multi-component HEAs were designed as equiatomic or near equiatomic and were mainly prepared by vacuum arc melting. Mechanical alloying (MA) is a widely used high-energy solid-state, non-equilibrium, topdown processing route for the synthesis of advanced nanocrystalline materials [9, 10]. It involves milling of elemental powders to achieve alloying at atomic scale. The extension of solid solubility with good homogeneity and room-temperature processing are the main advantages of MA over the casting route associated with segregation and inhomogeneity, especially with multicomponent systems with large differences in the melting points. Further, MA route also decreases the tendency to ordering and can easily lead to the formation of high entropy alloys in the nanocrystalline structural state [10]. However, the disadvantage of MA route is its large duration.

This work was supported by Ministry of Education and Science of Ukraine [project \# 2106] 
This study reports the structural evolution of equiatomic high-entropy AlNiCoFeCrTi alloy from elemental materials to metastable solid solution during shot-time mechanical alloying/mechanical activation (MA), and then to equilibrium phases during subsequent thermal annealing. The microhardness of as-received powders was also investigated.

\section{EXPEROMENTAL}

\section{A. Materials and Processing}

Equiatomic elemental blend of $\mathrm{Al}, \mathrm{Ni}, \mathrm{Co}, \mathrm{Fe}, \mathrm{Cr}$, Ti powders with purity higher than $99.0 \%$ and particle size of $\leq 50 \mu \mathrm{m}$ was chosen for experimentations. Powder blend was subjected to shottime mechanical alloying within 3 hours using high-energy planetary ball mill [9] and subsequent thermal annealing during $1 \mathrm{~h}$ at temperature $1200{ }^{\circ} \mathrm{C}$ to synthesize senary equiatomic AlNiCoFeCrTi high entropy alloy.

Milling of elemental powders was carried out in high energy planetary ball mill at $580 \mathrm{rpm}$ with ball to powder weight ratio of 10:1. Hardened steel vial and balls of $10 \mathrm{~mm}$ in diameter were used as a grinding media and petrol was used as a process controlling agent. The powder samples were extracted at intervals of $1 ; 2 ; 3 \mathrm{~h}$ during milling.

The $3 \mathrm{~h}$ ball milled alloy powder was then heat treated $1 \mathrm{~h}$ at the temperature $1200^{\circ} \mathrm{C}$ in vacuum.

\section{B. Structural Characterization}

The effects of milling duration $(1,2,3 \mathrm{~h})$ and subsequent annealing at temperature of $1200{ }^{\circ} \mathrm{C}$ of as-MA alloy on the structure and phase transformation were investigated by means of Rigaku Ultima IV X-ray diffractometer (XRD) with $\mathrm{Cu} \mathrm{K} \alpha$ radiation. The range of scanning angle $(2 \theta)$ was from $20^{\circ}$ to $120^{\circ}$. The scanning step was $0.04^{\circ}$ with an angular speed of the goniometer rotation of $2 \% \mathrm{~min}$ The crystallite size in the milled powders as well as after annealing has been calculated from the XRD peak broadening using peak profile analysis after eliminating the instrumental and strain contributions. The lattice parameters of solid solutions were calculated according to the positions of the gravitation centers of the diffraction profiles at large angles based on the Wulff-Bragg's condition.

A scanning electron microscope (SEM) equipped with an energy dispersive spectrometer (EDS) was used to obtain basic information concerning microstructural features and measure the chemical composition of materials. Prior to SEM observation feedstock powder was glued by conductive compound to plate for preparing a slice by standard metallographic technique with polishing by diamond slurry.

\section{Mechanical Testing}

Microhardness measurements of the powder alloy were performed using a conventional microhardness machine Digital Microhardness Tester MHV-1000 equipped by standard Vickers' pyramid. Microhardness numbers were determined under indentation loads not higher than $1.0 \mathrm{~N}$ and a duration time of 15 s. 10-20 measurements were made on each sample for an average.

\section{RESULTS AND DISCUSSION}

\section{A. Structure Evolution of Elemental Powders During Mechanical Alloying}

Fig. 1 shows the XRD patterns of the equiatomic AlFeNiCoCrTi powders prepared under different milling times. The pattern of $0 \mathrm{~h}$ exhibits peaks of all the pure elements in the initial mixture. It can be seen that as milling time increases, drastic decrement of diffraction intensity and peak broadening are observed. The peak intensity decreases dramatically in the early stages of milling after the powder was grinded for $1 \mathrm{~h}$. Obvious peak broadening can be observed and a lot of peaks become invisible with an increase in grinding time. The disappearance of diffraction peaks can be seen as the beginning of the solid solution formation. Only the most intense diffraction peaks can be clearly seen in the $2 \mathrm{~h}$ ball milled powder, which indicates the formation of the single phase solid solution with bcc structure ( $\beta$-phase). As the milling time reaches up to $3 \mathrm{~h}$, the diffraction peaks exhibit no change except for a minor broadening. The intensity decrement and peak broadening in the diffraction can be attributed to the formation of nanocrystalline and high lattice strain induced by severe plastic deformation during the MA processing $[10,11]$.

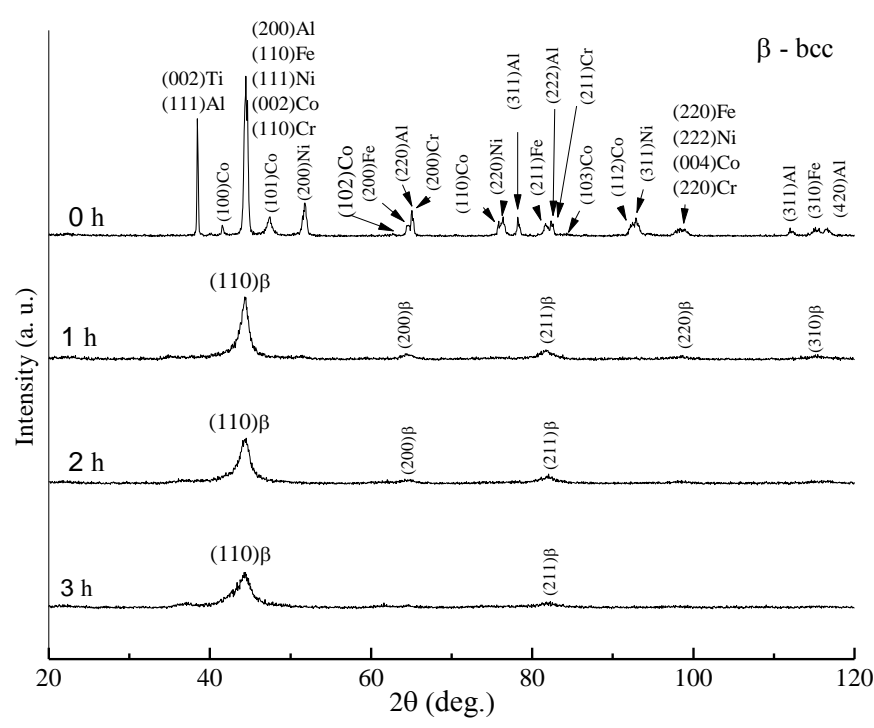

Fig. 1. XRD patterns of AlNiCoFeCrTi powders with different milling times

However, the cause of diffraction peak disappearance is complicated, which can be introduced by particle and crystal refinement together with high lattice strain, different atomic diffraction factor proportional to the atomic number, crystallinity decrement, formation of solid solution. For the XRD peak intensity, the distorted atomic planes increase $\mathrm{X}$ ray diffuse scattering effect and give smaller peak intensity [11]. Most of the diffraction peaks can hardly be seen after be ball milled for $3 \mathrm{~h}$. Thus, the formation of nanocrystalline and high lattice strain are primary factors of the above-mentioned broadening and decrement of diffraction intensity of the 
diffraction peaks. Moreover, size mismatch effect between the constituents, increasing grain boundary fraction and increasing dislocation density produced by severe plastic deformation lead to the increment of lattice strain [11].

The crystallite size of the alloy powders under different milling times have been calculated from the X-ray peak broadening using Scherrer's formula after eliminating the instrumental contribution. The crystallite size of the alloy powders after $2 \mathrm{~h}$ of milling was nearly $50 \mathrm{~nm}$. After $3 \mathrm{~h}$ of milling the crystallite size reduced below $36 \mathrm{~nm}$. The crystal structure of the nanocrystalline AlFeNiCoCrTi HEA is identified as bcc with lattice parameter $a=0.2886 \mathrm{~nm}$.

It is known that most of the binary metallic systems in the AlNiCoFeCrTi HEA system possess limited solid solubility under equilibrium condition because of their positive heats of mixing. However, the six components MA powders with milling duration higher than $1 \mathrm{~h}$ show simple solid solution structure, which can be attributed to the formation of supersaturated bcc solid solution. The solid solubility extension is introduced by the effect of high mixing entropy and the non-equilibrium state of the MA process. As the component number increases, the random diffusion between different elements will be enhanced and thus the solid solubility is extended. In addition, the high-entropy of mixing can lower the tendency to order and segregate, and therefore makes the solid solution more easily formed and more stable than intermetallics and other ordered phases [3,4]. On the other hand, the MA process can also extend the solid solubility limits $[9,10]$. Alloying occurs when the grain sizes of the elemental components decrease down to nanoscale and then a substantial amount of enthalpy can be stored in nanocrystalline alloy due to the large grain boundary area. The energy stored in the grain boundaries serves as the driving force for the formation of solid solution [11]. Interdiffusion among the components occurs and the solid solubility is expected to increase with milling time increasing until it reaches a supersaturation level, beyond which no further extension of solid solubility will be achieved $[9,10]$.

\section{B. Microstructure Characterization of MA AlNiCoFeCrTi High-Entropy Alloy}

It's known that the ball to powder interaction during highenergy ball milling involves continuous plastic deformation, fracture, and welding of particles finally leading to the nanocrystallization or even amorphization $[9,10]$. In the early period of MA process the particles firstly cold weld together to form even larger particles. The cold welded agglomerations are crushed down to smaller particles when the milling is prolonged. This circulation leads the powder be refined gradually and facilitates the diffusion and alloying among different metallic elements. The $3 \mathrm{~h}$ ball milled alloy powder shows refined morphology with average particle size of $35 \mu \mathrm{m}$ as exposed in Fig. 2. The results of quantitative elemental microanalysis on the EDX spectrum (not listed in this article) indicate that the homogeneity and equiatomic composition in each alloy particle after $3 \mathrm{~h}$ MA is not achieved.
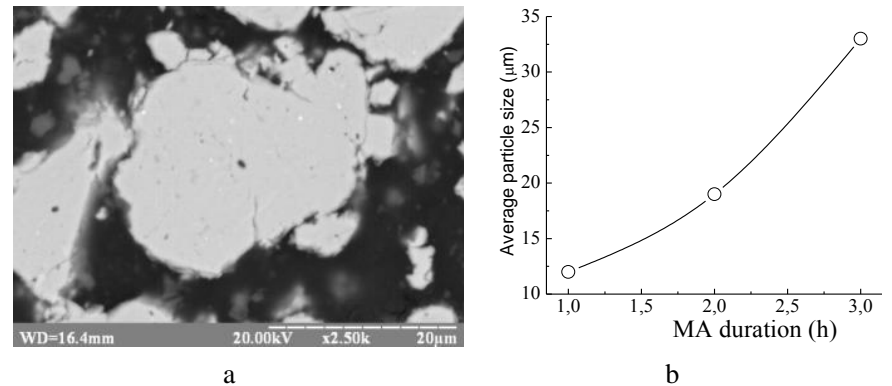

Fig. 2. SEM image of AlNiCoFeCrTi high-entropy alloy powder after $3 \mathrm{~h}$ milling duration (a) and changes of the powder particles size depending on the duration of mechanical alloying

\section{Structure Characterization of AlNiCoFeCrTi High-Entropy Alloy after Annealing}

After annealing for $1 \mathrm{~h}$ at the temperature $1200{ }^{\circ} \mathrm{C}$ the phase composition of the mechanically alloyed AlNiCoFeCrTi powder transforms to bcc and fcc solid solutions, as can be seen in Fig. 3. The results of XRD analysis identify that during thermal annealing precipitation of $\mathrm{TiC}$ and grain growth of equilibrium phases occur. The formation of $\mathrm{TiC}$ is associated with the high activity of $\mathrm{Ti}$, which is not completely dissolved in bcc solid solution under MA, and as a result, during annealing, $\mathrm{Ti}$ also reacts with carbon, which is a component of petrol, used as a process controlling agent. The final alloy showed a mixture of fcc and bcc phases with TiC precipitates.

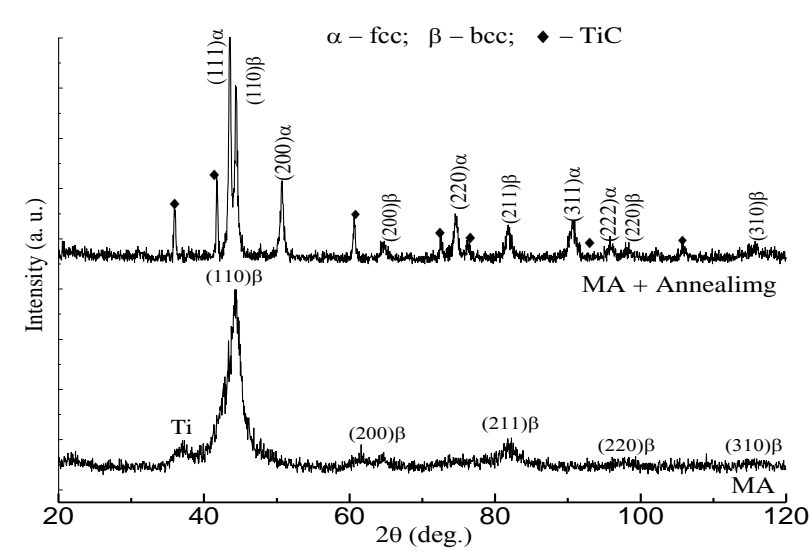

Fig. 3. XRD patterns of the $3 \mathrm{~h}$ MA AlNiCoFeCrTi high-entropy alloy powder after annealing for $1 \mathrm{~h}$ at temperature $1200{ }^{\circ} \mathrm{C}$

Simple equilibrium phases obtained in the annealed state of MA powders confirm that the high-entropy effect enhances the formation of simple solid solution phases instead of complex compound phases.

The phase transformation under annealing temperature can be attributed to the metastable state of the supersaturated bcc solid solution [8]. This metastable structure transforms during annealing to stable equilibrium fcc and bcc solid solutions, $\alpha$ and $\beta$ - phases, respectively.

The XRD pattern confirms that the as-annealed alloy is composed of major fcc phase and the less volume fraction of bcc phase. Obvious peak broadening, shown in Fig. 4, confirm 
the nanocrystalline nature of AlNiCoFeCrTi HEA with fcc and bcc structure. The crystallite size of the main fcc phase of the alloy is $47 \mathrm{~nm}$ and the lattice parameters of the phases present in the alloy are $0.3594 \mathrm{~nm}$ for $\alpha$-phase, $0.2884 \mathrm{~nm}$ for $\beta$-phase, respectively.

Nanocrystallinity of milled powders is retained in AlNiCoFeCrTi HEA even after heat treatment at elevated temperature. Presence of fine oxide and carbide particles together with slow rates of diffusion was believed to be responsible for high thermal stability of the alloy. The activation parameters for grain growth in spark plasma sintered (SPSed) $\mathrm{CoCrFeNi}$ and $\mathrm{CoCrFeMnNi}$ HEAs have been determined [10]. The activation energy (Q) values are 175 and $241 \mathrm{~kJ} / \mathrm{mol}$ for $\mathrm{CoCrFeNi}$ and $\mathrm{CoCrFeMnNi}$, respectively, which is in close agreement with the $\mathrm{Q}_{\mathrm{GB}}$ of $\mathrm{Ni}$ grain boundary diffusion in these HEAs [12]. This highlights the key role of grain boundaries in determining thermal stability of nanocrystalline HEAs.

SEM micrograph of AlNiCoFeCrTi HEA after annealing is shown in Fig. 4, which reveals the presence of three phases.

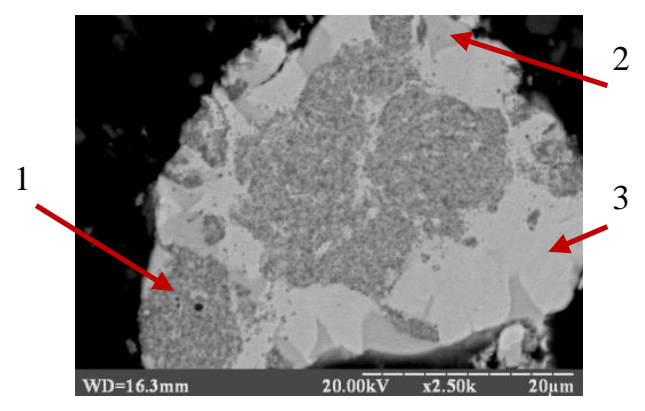

Fig. 4. SEM image of AlNiCoFeCrTi high-entropy alloy after annealing at temperature $1200^{\circ} \mathrm{C}$ during $1 \mathrm{~h}$

The results of quantitative elemental microanalysis on the EDX spectrum clearly indicate the homogeneity and equiatomic composition in each alloy particle after $1 \mathrm{~h}$ of annealing at temperature $1200^{\circ} \mathrm{C}$. The EDX chemical composition of the each phase present in the alloy is listed in Table. Grey phase ( 1 in Fig.4) has all the six elements and is rich in $\mathrm{Al}$, and $\mathrm{Ti}$ and depleted in $\mathrm{Cr}, \mathrm{Fe}, \mathrm{Co}$, and $\mathrm{Ni}$. Light grey phases (2 in Fig. 4) are rich in $\mathrm{Cr}$ and $\mathrm{Fe}$, and depleted in $\mathrm{Al}, \mathrm{Ni}$ and Ti. White phases (3 in Fig. 4) are rich in Co and Ni, and depleted in $\mathrm{Fe}, \mathrm{Cr}, \mathrm{Al}$ and $\mathrm{Ti}$.

TABLE Chemical Composition of Phases IN AlNiCoFeCrTi HighENTROPY ALLOY AFTER ANNEALING

\begin{tabular}{|c|c|c|c|c|c|c|}
\hline Phase & $\mathrm{Al}$ & $\mathrm{Ni}$ & $\mathrm{Co}$ & $\mathrm{Fe}$ & $\mathrm{Cr}$ & $\mathrm{Ti}$ \\
\hline Bulk & 16,6 & 16,7 & 16,7 & 16,8 & 16,6 & 16,6 \\
\hline 1 & 22,9 & 12,2 & 12,4 & 14,2 & 13,9 & 24,4 \\
\hline 2 & 14,1 & 14,2 & 15,9 & 22,6 & 20,7 & 12,5 \\
\hline 3 & 14,8 & 23,7 & 22,3 & 14,3 & 13,1 & 11,8 \\
\hline
\end{tabular}

\section{Mechanical Properties of AlNiCoFeCrTi High-Entropy Alloy Resulted from MA Process and Subsiquent Themal Annealing}

The Vickers microhardness, $H V$, of $3 \mathrm{~h}$ mechanically alloyed AlNiCoFeCrTi alloy is $7.1 \pm 0.25 \mathrm{GPa}$. The microhardness, $H V$, of $3 \mathrm{~h}$ milled alloy powder after annealing in vacuum at $1200{ }^{\circ} \mathrm{C}$ for $1 \mathrm{~h}$ increases and is $9.2 \pm 0.3 \mathrm{GPa}$. The high hardness could be attributed to the nanocrystalline nature of the alloy, the solid solution strengthening equiatomic nature of the solid solution phases, the presence of two/three phases structure $[3,4,6,8]$, and due to the appearance of $\mathrm{TiC}$ precipitations after thermal annealing. The high hardness of 9.2 $\mathrm{GPa}$ prove the promising future of the alloy. These AlNiCoFeCrTi HEA should also have good wear resistance according to the conclusion, that is, the wear resistance of materials is in general proportional to their Vickers hardness.

\section{CONCLUSIONS}

Shot-time mechanical ball milling for $3 \mathrm{~h}$ has been employed to synthesize nanocrystalline equiatomic AlNiCoFeCrTi HEA consisting of metastable supersaturated substitutional solid solution with bcc crystal structure. High mixing entropy decreases free Gibbs energy of equiatomic alloy, reducing the tendency to ordering and segregation, facilitates the formation of a solid solution, and increases its stability compared to intermetallics and other ordered phases. The maximized configurational entropy of HEAs is expected to result in simple solid solution structures. Furthermore, MA has been shown to extend solid solubility even in immiscible systems $[13,14]$. Therefore, the propensity of getting simple solid solution phases is higher during MA of HEAs. Although alloying is not complete at this milling time, it goes to completion during thermal annealing to achieve the alloy formation.

A detailed XRD study on mechanically alloyed highentropy AlNiCoFeCrTi alloy after thermal annealing at $1200{ }^{\circ} \mathrm{C}$ for $1 \mathrm{~h}$ revealed the formation of a three phase structure consisting of fcc and bcc solid solutions with fine precipitates of $\mathrm{TiC}$ (to $8 \%$ ). The alloy retains its nanocrystallinity even after heating to $1200{ }^{\circ} \mathrm{C}$. Presence of fine oxide and carbide particles together with slow rates of diffusion was believed to be responsible for high thermal stability of the alloy.

The powder AlNiCoFeCrTi HEA has high hardness due to solid-solution hardening and strong lattice distortion resulting from different atomic radii of substitutional elements, nanostructured state, and presence of $\mathrm{TiC}$ precipitations.

\section{References}

[1] Nanostructured High-Entropy Alloys with Multiple Principle Elements: Novel Alloy Design Concepts and Outcomes / J.W. Yeh, S.K. Chen, S.J. Lin and et. al. // Advanced Engineering Materials, 2004, 6: P. 299-303.

[2] B. Cantor, I.T.H. Chang, P. Knight, A.J.B. Vincent. Microstructural development in equiatomic multicomponent alloys Microstructural development in equiatomic multicomponent alloys // J. Mater. Sci. Eng. A, 2004, 375-377: 213-218. 
[3] B.S. Murty, J.W. Yeh and S. Ranganathan. High-Entropy Alloys. Elsevier, 2014, $218 \mathrm{p}$.

[4] High-Entropy Alloys. Fundamentals and Applications. Editors M. C. Gao , J.-W. Yeh, P. K. Liaw, Y. Zhang. Elsevier, 2015, 516 p.

[5] Yeh J. W. High-entropy alloys - a new era of explotation / J. W. Yeh, Y. L. Chen, S. J. Lin // Mater. Sci. Forum. - 2007. - Vol. 560. - P. 1-9.

[6]. Y.F. Ye, Q. Wang, J. Lu, C.T. Liu and Y. Yang. High-entropy alloy: challenges and Prospects // Materials Today, Volume 19, Number 6, July/August 2016, P. 349-362.

[7] Ming-Hung Tsai \& Jien-Wei Yeh . High-Entropy Alloys: A Critical Review // Materials Research Letters, 21 February 2017.

[8] D.B. Miracle, O.N. Senkov. A critical review of high entropy alloys and related concepts // Acta Materialia. 122 (2017) 448-511.

[9] C. Suryanarayana, "Mechanical alloying and milling", Prog. Mater. Sci., vol. 46, pp. 1-184, January 2001.

[10] Mayur Vaidya, Garlapati Mohan Muralikrishna, Budaraju Srinivasa Murty "High-entropy alloys by mechanical alloying: A review" /" Materials Research Society. January2019. pp. 664-686

[11] J.-W. Yeh, J., S.Y. Chang, Y.D. Hong and et. al., "Anomalous decrease in X-ray diffraction intensities of $\mathrm{Cu}-\mathrm{Ni}-\mathrm{Al}-\mathrm{Co}-\mathrm{Cr}-\mathrm{Fe}-\mathrm{Si}$ alloy systems with multi-principal elements", Mater. Chem. Phys., vol. 103, pp. 41-46, May 2007.

[12] M. Vaidya, K.G. Pradeep, B.S. Murty, G. Wilde, and S. V. Divinski: Radioactive isotopes reveal a non sluggish kinetics of grain boundary diffusion in high entropy alloys. // Sci. Rep.7,1 (2017)

[13] K.B. Zhang, Z.Y. Fu, J.Y. Zhang, et al., "Nanocrystalline CoCrFeNiCuAl high-entropy solid solution synthesized by mechanical alloying" // .J Alloys Compd, vol. 485, pp. L31-34, October 2009.

[14] B.S. Murty and S. Ranganathan: Novel materials synthesis by

mechanical alloying/milling // Int. Mater. Rev. 43, 101 (1998). 